\title{
Correction to: Hospice and End of Life Care in Neurologic Disease
}

Farrah N. Daly and Mara M. Lugassy

\section{Correction to:}

Claire J. Creutzfeldt, Benzi M. Kluger and Robert G. Holloway (eds.), Neuropalliative Care, https://doi.org/10.1007/978-3-319-93215-6_16

Chapter 16: Page page 224, the sentence in paragraph "Two physicians, usually the attending or referring physician..." has been updated as below:

"Two physicians, usually the attending or referring physician and the hospice medical director, must certify that they believe the patient's prognosis is 6 months or less if the disease takes a typical course." 